\title{
EFFECT OF TEMPFORMING TEMPERATURE ON THE MICROSTRUCTURE AND MECHANICAL PROPERTIES OF A HIGH-STRENGTH LOW-CARBON STEEL
}

\author{
Anastasiya DOLZHENKO, Zhanna YANUSHKEVICH, Kristina KOPTEVA, Andrey BELYAKOV, \\ Rustam KAIBYSHEV
}

Belgorod State University, Belgorod, Russian Federation,

dolzhenkoa@bsu.edu.ru

https://doi.org/10.37904/metal.2019.864

\begin{abstract}
The effect of tempforming temperature on the microstructure and mechanical properties, especially impact toughness, of the S700MC-type high-strength low-carbon steel was investigated. Tempforming at temperatures of 923 and $973 \mathrm{~K}$ was characterized by the development of ultrafine-grained layered microstructure consisting of grains highly elongated in the rolling direction with the transverse grain size of 530 and $920 \mathrm{~nm}$, respectively. This microstructure improved significant the mechanical properties including the toughness in a wide range of test temperatures. The steel tempformed at $923 \mathrm{~K}$ exhibited the ultimate tensile strength of $1110 \mathrm{MPa}$ and the impact toughness, $\mathrm{KCV}$, above $450 \mathrm{~J} / \mathrm{cm}^{2}$ at a temperature of $293 \mathrm{~K}$, and KCV of $109 \mathrm{~J} / \mathrm{cm}^{2}$ at a liquid nitrogen temperature for the impact direction perpendicular to the rolling plane. An increase in the toughness was achieved by absorbing the impact energy during the separation of steel in the direction perpendicular to the direction of impact.
\end{abstract}

Keywords: High-strength low-carbon steel, tempforming, ultrafine grained microstructure, delamination toughening, impact toughness

\section{INTRODUCTION}

High-strength low-carbon steels are workhorse materials in the most industries due to their low cost and good combinations of mechanical properties, such as strength, ductility and toughness. However, this type of steels with high strength typically exhibits low Charpy V-notch impact energy of 10 to $40 \mathrm{~J}$ at room temperature which is limits their structural applications [1]. These steels could not be used at temperatures below the ductilebrittle transition temperature (DBTT), at which the steel loses its toughness and fracture occurs in a brittle mode. It is known that the grain refinement is one of the most promising way to decrease DBTT concurrently with strengthening of structural steels and alloys $[2,3]$. The ultrafine grained structure decreases the stress concentration at grain boundaries, especially at triple junctions [3].

The present work deals with a new method of thermomechanical treatment of high-strength low-carbon steels. This results in the formation of an ultrafine-grained layered microstructure with a uniform distribution of dispersed nanoscale particles of secondary phases and leads to an increase of the impact toughness at low temperatures. Such thermomechanical treatment of high-strength low-carbon steels is referred to as tempforming, which includes quenching and tempering followed by rolling at tempering temperature and allows obtaining a promising combination of mechanical properties in low-alloyed steels [4]. In particular, the tempformed steels exhibit delamination phenomenon, which improves the toughness at low temperatures [5]. The dominating factors controlling the delamination toughening are considered to be the ultrafine grains, the grain shape and the $\langle 110\rangle \| R D$ fiber texture, which vary depending on the starting microstructure and the processing conditions, such as rolling temperature and rolling reduction $[4,6]$. The aim of the present work is to report the results of our current investigations on the microstructure and mechanical properties of the S700MC-type high-strength low-alloy steel subjected to tempforming at different temperatures. The paper is particularly focused on the effect of tempforming temperature on the fracture toughness of the steel over a range of temperatures from $293 \mathrm{~K}$ to $77 \mathrm{~K}$. 


\section{EXPERIMENTAL}

The S700MC-type high-strength low-alloy steel (Fe - 0.09C $-0.12 \mathrm{Si}-1.19 \mathrm{Cr}-1.55 \mathrm{Mn}-0.003 \mathrm{P}-0.005 \mathrm{~S}-$ $0.05 \mathrm{Nb}-0.025 \mathrm{Al}-0.05 \mathrm{Ti}-0.42 \mathrm{Mo}-0.09 \mathrm{~V}-0.003 \mathrm{~B}$, all in mass \%) was subjected to homogenization annealing followed by hot forging at a temperature of $1423 \mathrm{~K}$. Then the steel was quenched from $1373 \mathrm{~K}$ and tempered at $923 \mathrm{~K}$ for $1 \mathrm{~h}$. This sample is referred to as initial one. The tempforming included quenching from $1373 \mathrm{~K}$, tempering at temperatures of 923 or $973 \mathrm{~K}$ for $1 \mathrm{~h}$ and rolling at tempering temperature to a total strain of 1.5. The structural investigations were performed on the RD-ND sections (RD - rolling direction, ND - normal direction), using an Olympus GX71 optical microscope and a Quanta Nova Nano-sem 450 scanning electron microscope with an electron back scattering diffraction (EBSD) analyser incorporating an orientation imaging microscopy (OIM). The EBSD mappings were subjected to clean up procedure setting minimal confidence index of 0.1 . The samples for structural investigations were electro-polished using an electrolyte containing 10 $\%$ perchloric acid and $90 \%$ acetic acid at a voltage of $20 \mathrm{~V}$ at room temperature. The mean grain size was evaluated on the OIM micrographs as an average distance between high-angle boundaries with misorientations of $\theta \geq 15^{\circ}$.

The tensile tests were carried out at ambient temperature by using an Instron 5882 testing machine on specimens with gauge length of $12 \mathrm{~mm}$ and cross section of $3.0 \mathrm{~mm} \times 1.5 \mathrm{~mm}$. The tensile axis was parallel to the rolling direction (RD). Standard Charpy V-notch specimens were tested using an Instron $450 \mathrm{~J}$ impact machine (Model SI-1M) with an Instron Dynatup Impulse data acquisition system at temperatures ranging from 77 to $293 \mathrm{~K}$.

\section{RESULTS AND DISCUSSION}

\subsection{Microstructure characterization}

The deformation microstructures evolved in the studied steel during tempforming at different temperatures are shown in Figure 1. The initial microstructure is also shown in Figure 1a for a reference.
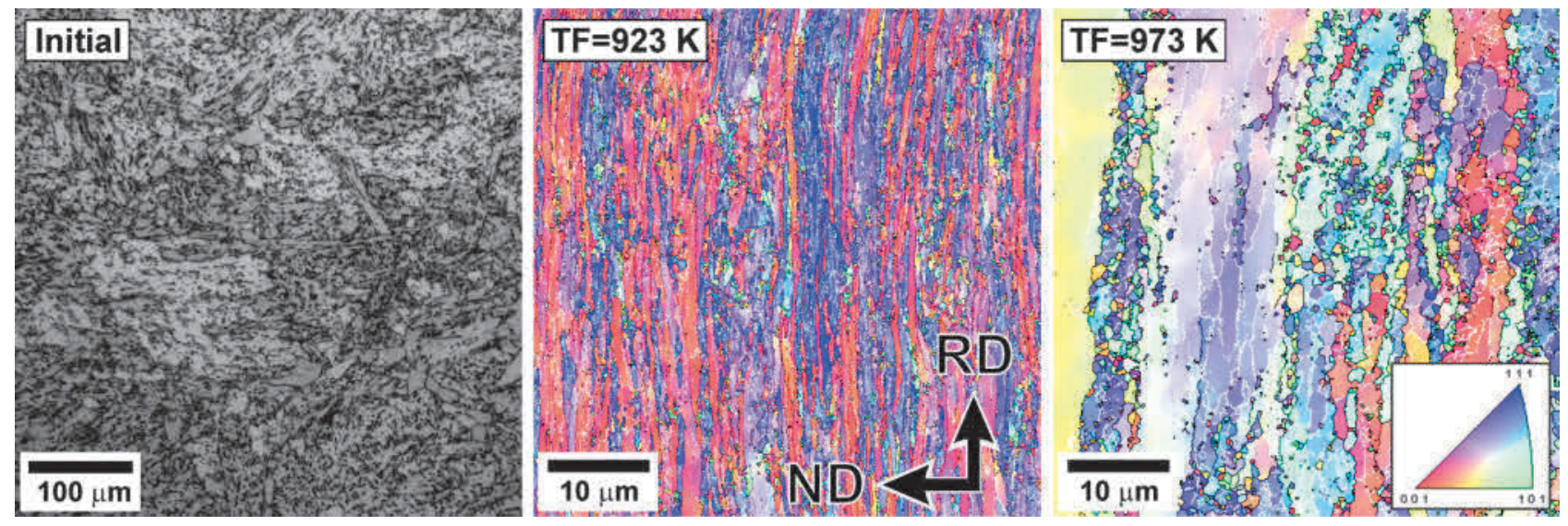

Figure 1 Typical microstructures of the S700MC-type high-strength low-carbon steel developed during initial treatment (a) and tempforming at $923 \mathrm{~K} \mathrm{(b)}$ and $973 \mathrm{~K}$ (c). The colours indicate the directions along ND

The microstructure that evolves during tempforming at a low temperature of $923 \mathrm{~K}$ is characterized by an elongation of grains along the rolling axis (Figure 1b). As a result, the developed microstructure consists of highly elongated ultrafine grains with the mean transverse grain size of $530 \mathrm{~nm}$. The investigated steel after tempforming at $923 \mathrm{~K}$ is characterized by strong $\langle 001\rangle|| N D$ and $\langle 111\rangle||$ ND fiber textures (corresponding to red and blue colours, respectively, in Figure 1b). It was shown that intensity of the $\{100\}\langle 110\rangle$ texture 
increases in low-carbon steels during multi-pass warm plate rolling at temperatures of 813 - $923 \mathrm{~K}$ [7]. The highest relative intensity of 5 was obtained for $\langle 001\rangle \|$ ND texture component. An increase in the tempforming temperature to $973 \mathrm{~K}$ results in an increase in the transverse grain size to $950 \mathrm{~nm}$ (Figure 1c). Similar to the microstructure developed by tempforming at $923 \mathrm{~K}$, the tempformed microstructure evolved at $973 \mathrm{~K}$ consists of highly elongated grains, which are interleaved with chains of the fine grains. Moreover, the warm deformation behaviour of the martensite blocks and/or packets strongly depends on their variations in the crystallographic orientation and their geometric arrangement, resulting in the evolution of a heterogeneous ultrafine grained microstructure [4, 8]. The microstructure of the steel during tempforming at $923 \mathrm{~K}$ is characterized by $\mathrm{Cr}_{23} \mathrm{C}_{6}$-type carbides with an average size of $50 \mathrm{~nm}$ precipitated along boundaries/subboundaries and homogeneously distributed $M(C, N)$ carbonitrides with an average size of 10 $\mathrm{nm}$ as clarified in previous study [9].

\subsection{Tensile properties and impact toughness}

Representative engineering stress-strain curves obtained by tensile test at ambient temperature for the steel in initial condition and subjected to tempforming at temperatures of 923 and $973 \mathrm{~K}$ are shown in Figure 2a; and the main tensile properties of the steel are listed in Table 1. Tempforming at $923 \mathrm{~K}$ resulted in remarkable strengthening of the steel. The tensile properties of the steel after tempforming at $923 \mathrm{~K}$ are characterized by high ultimate tensile strength and yield strength of 1100 and $1090 \mathrm{MPa}$, respectively.
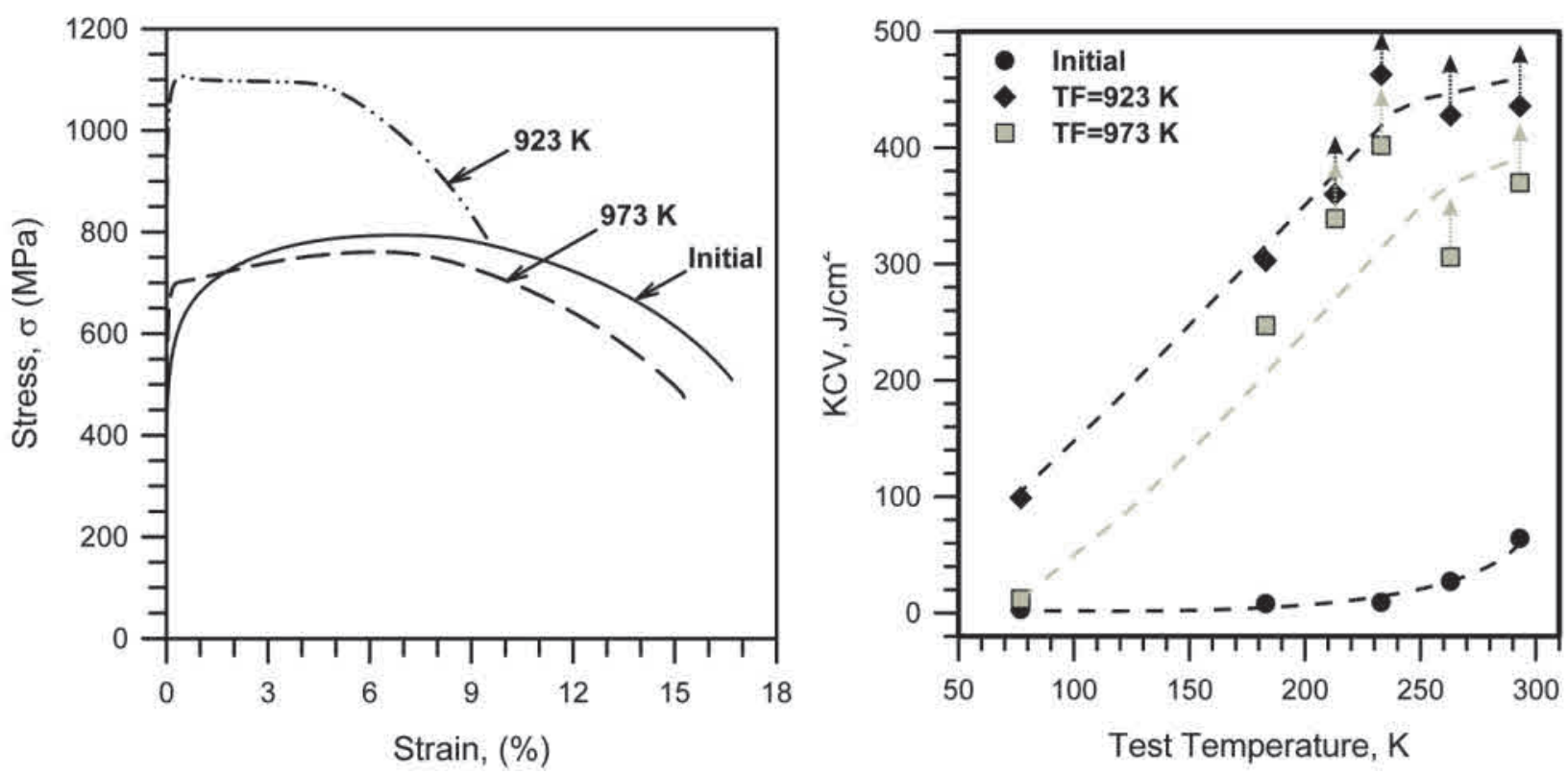

Figure 2 Room temperature tensile curves (a) and impact toughness at various temperatures (b) of the S700MC-type steel subjected to tempforming at different temperatures. The arrows indicate the nonfractured samples

An increase in the strength after tempforming is associated with the formation of an ultrafine grain layered microstructure. An increase in the strength after tempforming is accompanied by a decrease in total elongation to $9 \%$. Tempformed specimens at a temperature of $923 \mathrm{~K}$ are characterized by a negligible short stage of strain hardening after the onset of plastic flow during the tensile test that makes the ultimate tensile strength almost the same with the yield strength. An increase in tempforming temperature leads to significant change in the tensile behaviour. The ultimate tensile strength decreases to $760 \mathrm{MPa}$ and the total elongation increases to $15.3 \%$, which are comparable with initial condition. 
Table 1 Mechanical properties at ambient temperature of the S700MC steel subjected to tempforming at different temperatures

\begin{tabular}{|c|c|c|c|c|c|}
\hline & $\mathbf{K C V}_{\mathbf{2 9 3} \mathbf{K}, \mathbf{J} / \mathbf{c m}^{2}}$ & UTS, MPa & YS, MPa & Elongation, \% & HV \\
\hline Initial & 64 & 794 & 570 & 16.7 & 281 \\
\hline Tempforming at 923 K & 459 & 1100 & 1090 & 9.6 & 391 \\
\hline Tempforming at 973 K & 381 & 760 & 690 & 15.3 & 270 \\
\hline
\end{tabular}

The Charpy V-notch impact-absorbed energy of the S700MC-type steel subjected to tempforming at different temperatures is shown in Figure $\mathbf{2 b}$. The investigated steel subjected to tempforming at temperatures of 923 and $973 \mathrm{~K}$ exhibits substantially increased the impact toughness in a wide range of test temperatures. The samples tempformed at $923 \mathrm{~K}$ are characterized by larger impact toughness than those tempformed at $973 \mathrm{~K}$. This difference is about $100 \mathrm{~J} / \mathrm{cm}^{2}$ irrespective of test temperature from 77 to $293 \mathrm{~K}$. The impact toughness of the steel tempformed at $923 \mathrm{~K}$ reaches a maximum value at a test temperature of $233 \mathrm{~K}$, when $\mathrm{KCV}>463$ $\mathrm{J} / \mathrm{cm}^{2}$, followed by a decrease in the toughness with decreasing test temperature. The Charpy test specimens of the tempformed steel were not completely broken at $T \geq 233 \mathrm{~K}$ after impact tests. Therefore, the real values of the V-notch impact energy at these temperatures should be higher than indicated in Figure $\mathbf{2 b}$. The tempformed samples at $923 \mathrm{~K}$ exhibit superior delamination toughness. The high impact energy of $109 \mathrm{~J} / \mathrm{cm}^{2}$ is obtained even at liquid nitrogen temperature.

\subsection{Fractography}

The SEM images of impact fracture surfaces of the tempformed specimens after impact tests at $293 \mathrm{~K}$ and 77 $\mathrm{K}$ with corresponding Charpy $\mathrm{V}$-notched specimens after impact tests are shown in Figures $\mathbf{3}$ and $\mathbf{4}$, respectively.

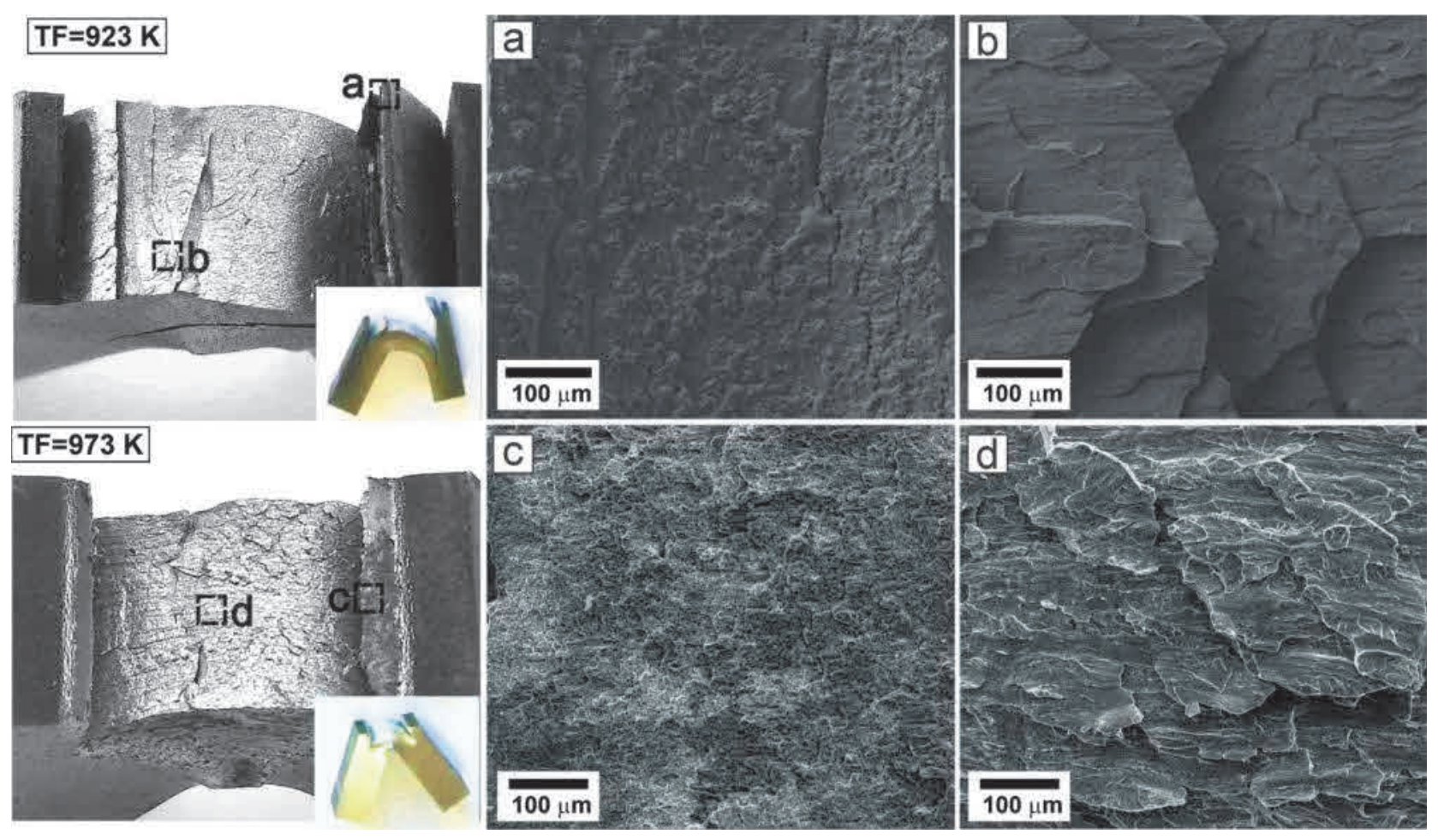

Figure 3 Fracture surfaces of the Charpy V-notch specimens of the steel tempformed at temperature of 923 $\mathrm{K}$ and $973 \mathrm{~K}$. The specimens were tested at $293 \mathrm{~K}$ 
The dimple fracture occurs during impact tests at $293 \mathrm{~K}$ in the samples subjected to tempfoming at $923 \mathrm{~K}$ on the planes normal to the delaminated layers (Figure 3a). Then, the stable crack propagation takes place that is associated with the delamination along RD-TD planes, i.e., crosswise to the impact direction, by transgranular cleavage (Figure $\mathbf{3 b}$ ). The fracture surface consists of almost flat terraces formed parallel to RD-TD plane. The delamination cleavage frequently initiates on parallel cleavage $\{100\}$ planes lying almost parallel to the rolling plane. An average dimension of terraces is about $200 \mu \mathrm{m}$. The stepwise terrace crack propagation belongs to crack arrester-type delamination [10]. The presence of elongated grains with $\langle 111\rangle \|$ $N D$ in the tempformed structure restricts the crack propagation along $\{100\}$ planes and promotes the crack branching. The sample tempformed at $973 \mathrm{~K}$ is characterized by an increase in the viscous fraction of fracture, which is especially noticeable on the planes normal to the delaminated layers (Figure 3c). Thus, a very high V-notch absorbed energy during the impact tests of the tempformed steel at different temperatures is attributed to the arrest of crack propagation in cleavage manner along the rolling plane and ductile fracture in perpendicular direction.

Decreasing the impact test temperature leads to anisotropy in the dimensions of delamination terraces (Figure 4). Their dimension along TD increases, while that in RD decreases. At $77 \mathrm{~K}$, the dimple fractures are observed in the local areas of initiation stage and tear ridges between the areas of quasi-cleavage fracture. At a test temperature of $77 \mathrm{~K}$ the tempformed steels exhibit zigzag crack propagation irrespective of of tempforming temperature (Figure 4). It is apparent that the zigzag crack propagation provides relatively high impact energy despite of hindering ductile fracture even at $77 \mathrm{~K}$. Even at liquid nitrogen temperature, the crack propagation crosswise to the impact specimen is suppressed. The Charpy specimens in the tempformed condition exhibit delaminations, i.e., the cracks branch along the impact test specimens, and the zigzagshaped cracks appear. The delamination is thought to relax triaxial stress conditions ahead of the advancing crack front and blunt the crack tip when the crack and crack branching planes intersect, thus leading to high impact energy [4]. A common feature in steels exhibiting similar delamination toughening behaviour is the stepwise crack propagation.
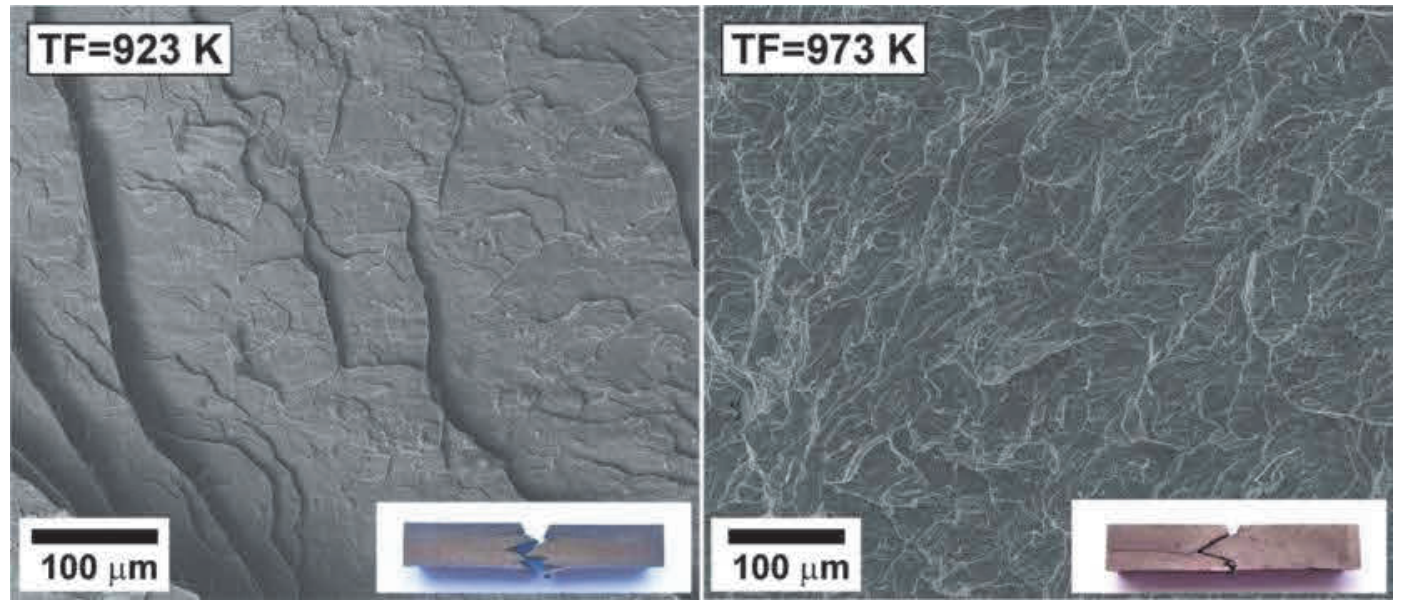

Figure 4 Fracture surfaces of the Charpy V-notch specimen of the tempformed steel at temperature of $923 \mathrm{~K}$ and $973 \mathrm{~K}$ tested at $77 \mathrm{~K}$

\section{CONCLUSION}

The new method of thermomechanical treatment of high-strength low-carbon steels, known as tempforming, is an effective way to improve the mechanical properties of S700MC high-strength low-carbon steel. The tempforming results in the development of an ultrafine grain structure with an average transverse grain size of 530 or $950 \mathrm{~nm}$ in steel subjected to tempforming at temperatures of 923 or $973 \mathrm{~K}$, respectively. Tempforming 
at $923 \mathrm{~K}$ results in remarkable strengthening of the steel and the strength increment increases with a decrease in the tempforming temperature. The ultimate strength of the steel subjected to tempforming at $923 \mathrm{~K}$ comprises to $1100 \mathrm{MPa}$. An increase in tempforming temperature leads to significant change in the tensile behavior of the investigated steel. The ultimate tensile strength decreases to $760 \mathrm{MPa}$ while total elongation remarkably increases. The tempformed steel exhibits substantially increased impact toughness in a wide range of test temperatures. The impact toughness of the samples tempformed at $923 \mathrm{~K}$ is about $100 \mathrm{~J} / \mathrm{cm}^{2}$ larger than that of the samples tempformed at $973 \mathrm{~K}$ in the whole range of test temperatures from 77 to $293 \mathrm{~K}$. The impact toughness of the steel tempformed at $923 \mathrm{~K}$ reaches a maximum value at a test temperature of $233 \mathrm{~K}$, when $\mathrm{KCV}>463 \mathrm{~J} / \mathrm{cm}^{2}$, followed by a decrease in the toughness to $109 \mathrm{~J} / \mathrm{cm}^{2}$ with decreasing test temperature to liquid nitrogen temperature. This high fracture toughness is attributed to the delamination, when the fracture occurs by cleavage along the rolling plane with large energy absorption.

\section{ACKNOWLEDGEMENTS}

The study was financially supported by the Russian Science Foundation, Project No. 17-72-10221. Authors are grateful to the staff of the Joint Research Center, "Technology and Materials", of the Belgorod State National Research University

\section{REFERENCES}

[1] TOMITA, Y. Development of fracture toughness of ultrahigh strength, medium carbon low alloy steels for aerospace applications. International Materials Reviews. 2000. vol. 45, pp. 27-37.

[2] TAKAKI, S., KAWASAKI, K. and KIMURA, Y. Mechanical properties of ultra-fine grained steels. Journal of Materials Processing Technology. 2001. vol. 117, pp. 359-363.

[3] SONG, R., PONGE, D. and RAABE, D. Mechanical properties of an ultrafine grained C-Mn steel processed by warm deformation and annealing. Acta Materialia. 2005. vol. 53, pp. 4881-4892.

[4] KIMURA, Y., INOUE, T. and TSUZAKI, K. Tempforming in medium-carbon low-alloy steel. Journal of Alloys and Compounds. 2013. vol. 577, pp. S583-S542.

[5] ZHOU, W. and LOH, N.L. Effect of delaminations on improvement of notch toughness at low temperatures. Scripta Materialia. 1996. vol. 34, pp. 633-639.

[6] KIMURA, Y., INOUE, T., Fuxing, Y. and TSUZAKI, K. Delamination toughening of ultrafine grain structure steels processed through tempforming at elevated temperatures. ISIJ International. 2010. vol. 50, pp. 152-161.

[7] BOURELL, D. Cleavage delamination in impact tested warm-rolled steel. Metallurgical Transactions A. 1983. vol. 14, pp. 2487-2496.

[8] LUGOVSKAYA, A., YANUSHKEVICH, Z., ODNOBOKOVA, M., BELYAKOV, A. and KAIBYSHEV, R. Effect of thermomechanical treatment on microstructure and mechanical properties of high-strength low-alloy steel. AIP Conference Proceedings. 2017. vol. 1909, no. 020120.

[9] DOLZHENKO, A., YANUSHKEVICH, Z., NIKULIN, S.A., BELYAKOV, A. and KAIBYSHEV, R. Impact toughness of an S700MC-type steel: Tempforming vs ausforming. Materials Science and Engineering A. 2018. vol. 723, pp. 259-268.

[10] KIMURA, Y. and INOUE, T. Combined effect of ausforming and warm tempforming on the strength and toughness of an ultra-high strength steel. ISIJ International. 2016. vol. 56, pp. 2047-2056. 